\title{
Observation of huge nonlinear absorption enhancement near exciton resonance in $\mathbf{G a N}$
}

\author{
Kung-Hsuan Lin, Gia-Wei Chern, Yin-Chieh Huang, and Chi-Kuang Sun \\ Graduate Institute of Electro-Optical Engineering ,National Taiwan University, Taipei, 10617, Taiwan \\ Phone 886-2-23659703, FAX 886-2-23677467, E-mail f0941009@ee.ntu.edu.tw \\ Stacia Keller, Umesh Mishra, and Steven P. DenBaars \\ Department of Electrical and Computer Engineering. University of California, Santa Barbara, CA 93106
}

\begin{abstract}
Huge nonlinear-absorption enhancement was observed in GaN system at the excitonic transition wavelength. This excitonic enhancement is attributed to four-wave-mixing type nonlinear processes. The peak nonlinear coefficient is at least $3000 \mathrm{~cm} / \mathrm{GW}$ corresponding to an enhancement factor of $>200$. The dephasing time of the exciton was also observed to be $60-160 \mathrm{fs}$ in $\mathrm{GaN}$ thin film at room temperature.

(C)2003 Optical Society of America

OCIS codes: 190.4720 Optical nonlinearities of condensed matter; 190.4180 Multiphoton processes.
\end{abstract}

\section{Introduction}

In this paper, we report the first observation of huge resonance of TPA coefficient near exciton resonance in semiconductor ever. This excitonic enhancement is not just attributed to four-wave-mixing (FWM) type nonlinear processes, but also due to the large exciton binding energy in GaN.

\section{Sample and Experimental setup}

To study the TPA with the excitation near the bandgap, we performed UV pump-probe experiments on the $2.5 \mu \mathrm{m}$ thick undped GaN and observed the significant enhancement of TPA.

\section{Experimental results}

Fig. 1 shows the typically measured probe transmission change $\Delta \mathrm{T} / \mathrm{T}$ of the nominally undoped GaN thin film as a function of probe delay at different central wavelengths near the exciton resonance photoexcitation. The gaussianlike component of the traces at zero time delay is contributed by the TPA process. The traces also reveal that the processes of excitons ionization and free carriers thermalization were $100-250 \mathrm{fs}$ and $600-800 \mathrm{fs}$, respectively. The detail of the fast carriers dynamics were discussed in our previous studies [1]. The magnitude of the TPA component is obviously enhanced near the photoexcitation of $362 \mathrm{~nm}$, which is very close to the exciton excitation energy in our sample.

\section{Discussion}

By assuming the pulse is gaussian-shaped in time and the beam is also gaussian-shaped in space, a TPA coefficient value $\beta$ can be estimated as

$$
\beta \approx-\frac{\Delta T}{T} \sqrt{\frac{\pi}{2 \ln 2}} \frac{\alpha}{I_{e}}
$$

$\alpha$ is the linear absorption coefficient and $I_{e}$ is the effective intensity of the pulse. The TPA coefficient values with different central wavelengths are showed in Fig. 2. For example, at a wavelength of $\sim 362 \mathrm{~nm}$, a TPA coefficient value of $2900 \mathrm{~cm} / \mathrm{GW}$ can be obtained, which is much higher than the already high below-bandgap TPA coefficient value of $15 \mathrm{~cm} / \mathrm{GW}$ at $400 \mathrm{~nm}$ [2].

To explain the enhancement of the TPA coefficient values near exciton resonance frequency, we considered a simplified three levels system. The system is initially in the ground state $|g\rangle$ with no excitons, and the final state $|f\rangle$ is the elctron-hole pair state. For the intermediate states, we assume that the contribution of the bandtail states is small and only take the exciton state $|n\rangle$ into consideration. The relation between the TPA coefficient and the photoexcitation frequency could be derived as 


\section{QTuJ1}

$$
\beta \propto \frac{1}{\left(\omega-\omega_{n g}\right)^{2}+\Gamma_{n g}^{2}},
$$

where $\omega_{n g}$ is the exciton resonance frequency and $\Gamma_{n g}$ is the dephasing term. The fitting results are also shown in fig. 2. An exciton dephasing time $\sim 66 \mathrm{fs}$ can be obtained. If we use a Lorentzian function convolved by the laser spectrum, the dephasing time is $\sim 155 \mathrm{fs}$. This results show that the room temperature dephasing time of excitons is close to the exciton ionization time of $\sim 200 \mathrm{fs}$.

\section{Summary}

We have observed the enhancement of nonlinear absorption near exciton resonance in GaN thin films using femtosecond pump-probe techniques. The TPA coefficient values of $\mathrm{GaN}$ near exciton resonance are at least 3000 $\mathrm{cm} / \mathrm{GW}$, and the dephasing time is 60-160 fs at room temperature. Detailed analysis and discussion will be presented in the talk.

\section{References}

[1] Y. - C. Huang, et al., "Femtosecond dynamics of exciton bleaching in bulk GaN at room temperature," Appl. Phys. Lett. 81, 85 (2002)

[2] C. -K. Sun, et al., "Two-photon absorption study of GaN," Appl. Phys. Lett. 76, 439 (2000).

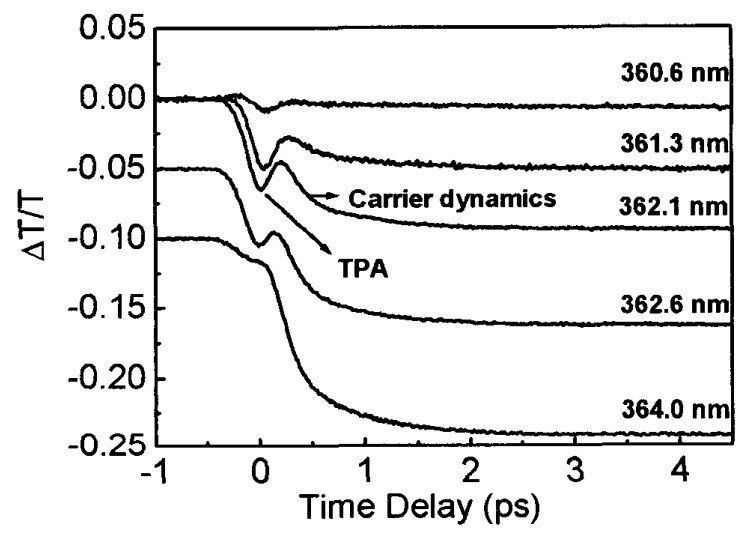

Fig. 1. Measured probe transmission transient response of the nominally undoped GaN thin film as a function of probe delay at different central wavelengths near the exciton photoexcitation. 


\section{QTuJ1}

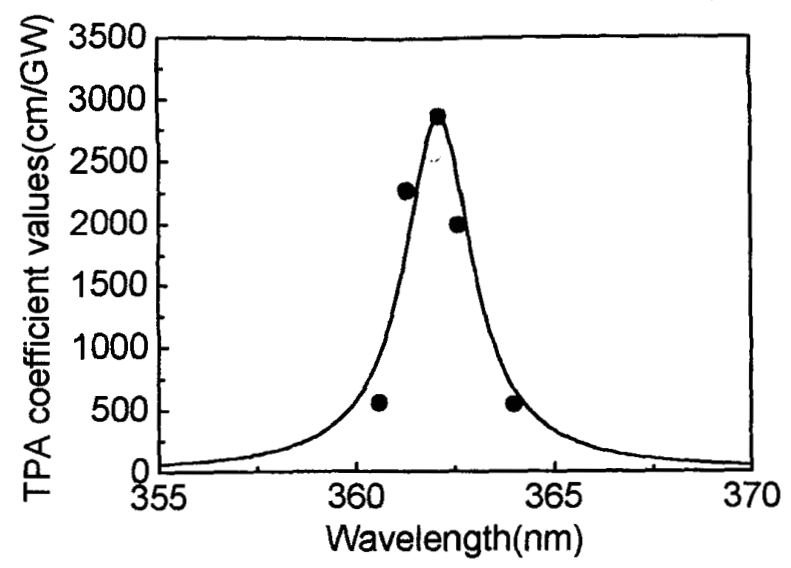

Fig. 2. The solid circles are the TPA coefficients calculated from the measured traces near the exciton resonance, and the solid line is the Lorentzian fitting curve. 